ESJ Humanities

\title{
La Résilience chez des Adolescentes Victimes d'agressions Sexuelles Commises par des Femmes en Côte d'Ivoire
}

\author{
Mélissa Affoué Kouassi
}

Assistante, Laboratoire de Psychologie Génétique

Différentielle/Département de Psychologie/Sciences de l'Homme et de la

Société (SHS) Université Félix Houphouët-Boigny, Côte d'Ivoire

\section{Anna-Corinne Bissouma}

Pédopsychiatre, Docteur en Psychologie, Chargée de Recherche à l'Institut National de Santé Publique, Côte d'Ivoire

Doi:10.19044/esj.2021.v17n36p129

Submitted: 20 September 2021

Accepted: 20 October 2021

Published: 31 October 2021
Copyright 2021 Author(s)

Under Creative Commons BY-NC-ND

4.0 OPEN ACCESS

Cite As:

Kouassi M.A. \& Bissouma A-C. (2021). La Résilience chez des Adolescentes Victimes d'agressions Sexuelles Commises par des Femmes en Côte d'Ivoire. European Scientific Journal, ESJ, 17 (36), 129. https://doi.org/10.19044/esj.2021.v17n36p129

\section{Résumé}

L'objectif de cette étude menée sur trois adolescentes en foyer d'accueil, est de comprendre le processus de résilience à travers des facteurs individuels, chez des victimes d'agressions sexuelles commises par des femmes, à Abidjan (Côte d'Ivoire). L'Hypothèse postule que ces facteurs sont déterminants dans la reconstruction résiliente. La méthode clinique et l'étude de cas ont guidé le choix des instruments de collecte des données, à savoir : le CD-RISC de Connor et Davidson (2003) et l'Exercice de résilience de Strümpfer (2001). La théorie des facteurs de protection de Garmezy (1990) a permis d'interpréter les résultats par le biais de l'analyse qualitative du contenu des réponses. Ces résultats montrent que les facteurs individuels interviennent dans la construction résiliente. Ceux-ci identifient la compétence cognitive, la spiritualité et la confiance en soi comme facteurs de protection de la résilience chez les participantes à l'étude.

Mots-clés : Résilience-Agression sexuelle-Femmes-Adolescentes 


\title{
Resilience in Adolescent Girls Victims of Sexual Assault by Women In Ivory Coast
}

\author{
Mélissa Affoué Kouassi \\ Assistante, Laboratoire de Psychologie Génétique \\ Différentielle/Département de Psychologie/Sciences de l'Homme et de la \\ Société (SHS) Université Félix Houphouët-Boigny, Côte d'Ivoire \\ Anna-Corinne Bissouma \\ Pédopsychiatre, Docteur en Psychologie, Chargée de Recherche à l'Institut \\ National de Santé Publique, Côte d'Ivoire
}

\begin{abstract}
The objective of the study on three adolescent girls in foster home, is to understand the process of resilience through individual factors, in victims of sexual assault committed by women, in Abidjan (Ivory Coast). The hypothesis postulates that these are determinants in resilient reconstruction. The clinical method and the case study guided the choice of data collection instruments, namely: the CD-RISC of Connor and Davidson (2003) and the Résilience Exercise of Strümpfer (2001). Garmezy's protective factor theory allowed us to interpret the results through qualitative analysis of the content of responses. These results show that individual factors are involved in resilient construction. They identify cognitive competences, spirituality and self-confidence as protective factors for resilience in the study participants.
\end{abstract}

Keywords: Resilience-Sexual assault-Woman-Adolescent girl

\section{Introduction}

Depuis les deux dernières décennies, les connaissances dans le domaine de l'agression sexuelle n'ont cessé de s'accroître, d'évoluer et de s'étendre à plusieurs disciplines. Les courants d'influence sont multiples et les retombées s'observent tant par les réformes de certaines législations et procédures judiciaires, que par des théorisations, des projets de recherche, ainsi que des pratiques cliniques adaptées et standardisées. Il en résulte qu'il y a une difficulté certaine à conceptualiser, expliquer voire décrire une agression sexuelle. En effet, ce thème alimente de nombreux débats et controverses au sein de la communauté scientifique (Bryden \& Grier, 2011). Ceux-ci concernent notamment les différents termes et définitions proposés qui varient considérablement, incluant violence sexuelle, viol, et coercition sexuelle.

Généralement, l'agression sexuelle fait référence aux contacts sexuels sans le consentement de la victime (Luissier, 2017). Ainsi, contrairement au 
viol, elle n'est pas limitée aux situations où l'agresseur pénètre ou tente de pénétrer sa victime. Elle n'est pas non plus limitée aux situations où l'agresseur utilise la violence physique afin de contraindre la victime. Toutefois, elle ne devrait pas être réduite à un contact physique transgressant le respect de l'intimité corporelle du sujet, qu'il y ait ou non relation sexuelle (Manciaux, 2005). Il pourrait donc en exister des formes sans autres contacts, telles que : l'exhibitionnisme, l'utilisation perverse du regard, l'exposition des enfants aux ébats sexuels d'adultes par média interposé ou non. Cette auteure affirme également qu'il y a agression psychologique sexualisée lorsqu'un enfant est manipulé par un adulte pour alléguer des agressions sexuelles qui n'existent pas.

Certaines définitions de l'agression sexuelle peuvent sembler restreintes, d'autres (très contestées) paraissent larges et englobent toutes situations. Aussi, pouvons-nous retenir que l'agression sexuelle est un acte à caractère sexuel, avec ou sans contact physique, commis par un individu sans le consentement de la personne visée, ou dans certains cas, notamment celui des enfants et des adolescents, par une manipulation affective ou par du chantage. Elle peut ainsi se manifester sous différentes formes: attouchements, viol ou harcèlement sexuel et a des conséquences sur la santé physique et mentale des victimes, particulièrement chez les mineures dont la trajectoire développementale semble affectée. Sur le plan psychologique, elle est un traumatisme qui entraîne la confusion, la perte de repère, le sentiment d'impuissance, le choc ou chaos émotionnel. En effet, comme tout traumatisme, elle peut avoir pour conséquences un trouble (chronique) de stress post-traumatique. D'une façon générale, elle peut "propulser» l'individu dans toute une symptomatologie signant les traces du trauma. Mais, singulièrement, chez les victimes mineures, les conséquences convergent vers la mise en évidence d'une vulnérabilité psychologique définitive (Pascal \& Hie, 2004). L'effet traumatique de l'agression sexuelle est potentiellement déstructurant et souvent, sur mineur, elle s'accompagne du secret et du refoulement psychique. Ainsi, le sentiment de culpabilité peut être amplifié par l'injonction du secret imposé.

Pendant l'adolescence, la dépression et le risque de suicide sont parfois inhérents aux blessures traumatiques causées par l'agression sexuelle (Heim et al, 2000). En tenant compte du fait que cette période spécifique est faite de bouleversements hormonaux, de questionnement sur l'identité et sur la sexualité, où les changements physiques entraînent des bouleversements psychologiques, notamment au niveau de l'estime de soi et du rapport à l'autre, la survenue d'une agression sexuelle vient potentialiser les problématiques. De ce fait, le concept d'agression sexuelle à l'adolescence revêt une forme particulière. Soutoul (1994) révèle que les victimes sont majoritairement des filles. Lagrange (1995) quant à lui affirme qu'un tiers des 
victimes affirme avoir subi des violences de manière répétée, principalement dans un contexte extrafamilial, de la part d'un homme connu de la même tranche d'âge. Par contre, 9\% des agressions sexuelles surviennent dans un contexte intrafamilial (Gabel, 2002).

Si les hommes sont le plus souvent en cause, les agressions sexuelles sont aussi le fait de femmes. Le phénomène des agressions sexuelles commises par les femmes est connu comme étant plutôt rare (Saradjan, 2010). Peu d'études ont été faites sur le sujet et ne concernent généralement que des agressions sexuelles commises par des femmes sur des individus de sexe masculin. En effet, l'étude de Freeman et Sandler (2008) a davantage les similitudes que les différences entre les agresseurs des sexes féminin et masculin. La principale différence concerne une probabilité plus élevée d'agresser sexuellement des garçons. Selon les auteurs, les délits perpétrés par les femmes consistent en attouchements sexuels, masturbations, contacts bucco-génitaux, pénétrations digitales et rapports sexuels avec pénétration. Il importe de retenir que dans cette étude, $50 \%$ des femmes ont commis leur délit avec un co-accusé (souvent un partenaire sexuel de sexe masculin).

De façon générale, la banalisation ou la sous-estimation des agressions sexuelles perpétrées par les femmes continue de soulever la question de la fiabilité des résultats en comparaison avec un tableau réel. Les représentations du rôle de la femme, reliées aux constructions sociales et culturelles, maintiennent son rôle nourricier, protecteur et non-agresseur en lien avec la sexualité ; ce qui influencerait négativement la détection et le dévoilement des agressions sexuelles commises par les femmes (Freeman \& Sandler, 2008 ; Saradjan, 2010). Les femmes qui ont agressé sexuellement de jeunes enfants sont souvent non judiciarisées. Au Canada par exemple, elles sont dirigées vers des services d'aide ou de traitement (Cortoni et al., 2009).

En Afrique, la recherche sur les abus sexuels est peu documentée (Mbassa, 2009) dans un contexte culturel où, d'une part la sexualité est taboue et d'autre part le recours à des punitions corporelles jointes à des insultes fait partie de l'éducation des enfants dont la voix n'est pas entendue et la parole niée; d'où le fait que la dénonciation demeure difficile. En Côte d'Ivoire, les questions relatives à la sexualité constituent également et toujours un tabou. Le rapport VACS sur les violences faites aux enfants et aux jeunes, publié en mars 2020 , montre que trois filles sur cinq $(58,0 \%)$ et deux garçons sur trois $(66,5 \%)$ ont été victimes de tout type de violence, pendant leur enfance. De plus une fille sur cinq $(19,2 \%)$ et un garçon sur dix $(11,4 \%)$ ont été victimes de violence sexuelle avant l'âge de 18 ans. Environ la moitié des filles (46,3\%) et des garçons $(45,7 \%)$ victimes de violences sexuelles durant l'enfance n'en n'ont jamais parlé à qui que ce soit. Parmi les victimes, rares sont celles qui ont fait appel à des services de prise en charge. Les filles /femmes qui ont subi des violences sexuelles ou émotionnelles pendant leur enfance sont plus 
susceptibles de souffrir de détresse mentale (76,4\% et 56,3\%, respectivement) que celles qui n'en n'ont jamais subi (Rapport VACS-CI, 2020).La typologie professionnelle des agresseurs est rapportée mais le sexe n'est pas pris en compte. Cela laisse sous-entendre que les agresseurs sexuels sont du sexe opposé à celui de la victime. Le rapport relève par ailleurs l'insuffisance de dénonciation des violences sexuelles et de prise en charge.

Ainsi, dans la société ivoirienne, «à cheval» entre tradition et modernisme, où la culture garde l'empreinte de valeurs et d'ordonnances mystico-religieuses, la problématique de l'agression sexuelle demeure équivoque (Dagou \& Goin Bi, 2015). La pratique des mutilations génitales en est un exemple. Elle est reconnue par la législation ivoirienne comme une violence envers les femmes, depuis 1998. De ce fait, c'est une pratique punie par la loi. Bien que de moins en moins répandue, elle demeure pourtant, sacrée et valorisante en termes d'initiation, dans certaines villes du centre ouest (Koudou et al., 2015). C'est une ambiguïté à laquelle les études sur les agressions sexuelles de femmes commises par des femmes doivent faire face en Côte d'Ivoire. Certaines agressions peuvent passer pour des pratiques religieuses, d'autres par contre, relèvent de relations homosexuelles, qui sont encore taboues. Or, ces dernières représentent environ 5\% des délits commis à travers le monde (Tardif et al., 2005).

Les agressions sexuelles d'adolescentes commises par des femmes ont des conséquences analogues pour la victime, à celles consécutives aux agressions commises par les hommes (Manciaux, 2005). Toutefois, d'un point de vue culturel, on pourrait s'attendre à ce que dans une société traditionnaliste comme celle de la Côte d'Ivoire où les relations sexuelles entre personnes du même sexe sont interdites, voire punies par la loi, le trouble de stress posttraumatique (TSPT) revête une forme différente. Les risques de rejet par le milieu familial, d'être «traité » d'homosexuel, ou l'éventualité d'être poursuivi en justice, sont des facteurs spécifiques qui donneraient une nuance particulière au TSPT. En l'occurrence, des symptômes comme les troubles émotionnels, la confusion et le désarroi chez l'adolescente s'intensifient. À cela s'ajoute un flou entre norme sexuelle et identité sexuelle, comme le décrit l'étude de Le Pape (2013) sur les viols d'hommes commis par des hommes. En effet, cette étude a montré qu'en plus des symptômes liés aux troubles posttraumatiques, la question de la «masculinité » se pose. En d'autres termes, les victimes ont le sentiment individuel de perte du statut masculin. S'il est indéniable que cette étude concerne uniquement les viols d'hommes commis par des hommes, elle a la particularité de donner des indications sur l'état de santé mentale des victimes d'agression homosexuelle.

C'est fort de cela que nous imaginions faire la rencontre avec le trauma, pendant l'étude: un évènement dramatique, avec des circonstances « aggravantes », corrélé à une symptomatologie atypique. La réalité clinique 
fût toute autre, notamment lors de la rencontre avec les adolescentes victimes d'agressions sexuelles commises par des femmes à Abidjan. En dépit des risques de désorganisation psychique qui surviennent après un évènement traumatique aussi atypique, nous avons constaté, chez certaines victimes, un développement normal, sans symptômes, ni problème d'adaptation et/ou de santé mentale. Dans la littérature scientifique, un tel tableau clinique fait référence à la résilience. C'est un construit décrit par Cyrlnick (1999), qui consiste pour un individu affecté par un traumatisme, à se reconstruire d'une façon socialement acceptable. L'approche de la résilience considère l'individu à partir des potentiels de résistance et de rétablissement, en tant que sujet capable de trouver face à l'adversité des réponses adaptatives variées qui lui permettent de se construire malgré, ou à partir des situations délétères ou traumatogènes. Dans cette perspective, du fait des différences individuelles, face à des facteurs de risque identiques, certains individus sont moins susceptibles que d'autres de développer des troubles psychopathologiques.

La résilience chez les victimes d'agression sexuelle est un sujet déjà abordé dans la littérature scientifique. Dans une étude de Bénoudji et al. (2017) réalisée au Tchad, les auteures affirment que la résilience après des violences sexuelles liées aux conflits armés dépend de la capacité à s'adapter, se transformer, ou développer des actions protectrices. Sadia et Kouakou (2019), quant à eux, attestent que les déterminants personnels et sociaux de résilience favorisent la reconstruction identitaire et la participation sociale des enfants victimes d'abus sexuels. Ouatara et al. (2014), ainsi que Kouakou-Mlan (2019), avancent la thèse des différences individuelles, selon laquelle, malgré des circonstances difficiles, certaines personnes arrivent à se protéger et à construire une vie positive. La présente étude tente d'élucider la question de la généralisation des travaux de ces chercheurs. Plus précisément, il est question de savoir s'il est possible de faire les mêmes observations sur des adolescentes relativement à d'autres formes de traumatisme atypique tel que les agressions sexuelles commises par des femmes. Au demeurant, nous privilégions les facteurs intra-individuels.

Des auteurs comme Anthony (1982), Garmezy (1985) et Ruter (1990) ont initié des recherches sur les enfants à risque, révélant que ceux-ci sont inégaux devant le risque et présentent un état de vulnérabilité variable. Ces différences interindividuelles s'expliqueraient par la faculté à mettre « en jeu » des capacités de résistance et de protection : le tempérament, les aptitudes cognitives, l'estime de soi, l'humour, l'empathie. Dans ce contexte, la résilience suppose la mobilisation de compétences, malgré une exposition à un stress sévère.

Au-delà de la recherche des facteurs individuels susceptibles d'être des facteurs de résilience, cette étude tente d'établir un lien entre ceux-ci et la résilience chez trois adolescentes victimes d'agressions sexuelles, commises 
par des femmes. Dans la mesure où l'impact traumatique semble différent chez chaque individu, il paraît opportun d'étudier les mécanismes impliqués dans la reconstruction du sujet. De manière spécifique, l'objectif de cette étude est d'analyser les déterminants intra-individuels favorisant la résilience chez des adolescentes victimes d'agressions sexuelles. L'hypothèse générale postule que les facteurs de protection intra-individuels permettent une reconstruction résiliente de l'individu. L'étude s'appuie sur la théorie de Garmezy (1990) qui stipule que les facteurs personnels favoriseraient la résilience. En effet, elle a identifié la réussite scolaire, un comportement adapté en classe, des aptitudes interpersonnelles (sociabilité, empathie, attractivité, humour, confiance en soi, contrôle), comme des indicateurs de résilience. Ces facteurs révèlent des modes d'adaptation et de résistance, en particulier chez l'enfant marqué par des conditions de vie délétères et une adversité importante. Les instruments de mesure utilisés sont le Connor et Davidson Resilience Scale ou CD-RISC (2003) qui permet d'évaluer les facteurs de protection concourant à la résilience. Cette échelle a été conçue pour répondre uniquement à l'évaluation des variables individuelles. À cela s'ajoute, le test de l'Exercice de résilience (Strümpfer, 2001), qui est un test projectif créé pour évaluer spécifiquement la résilience.

\section{Méthodologie}

\subsection{Site et participantes à l'enquête}

L'étude s'est déroulée auprès de six adolescentes. Cependant, seulement trois ont été sélectionnées en fonction des scores et réponses obtenus aux différents tests. Elles sont âgées respectivement de 13, 15 et 17 ans. Elles sont, par ailleurs, prises en charge dans un foyer tenu par une congrégation religieuse à Abidjan (Côte d'Ivoire). Ce lieu de vie accueille des jeunes filles itinérantes, sans logis. Compte tenu des poursuites judiciaires (les relations homosexuelles sont illégales en Côte d'Ivoire), auxquelles elles pourraient s'exposer, le site exact de l'enquête demeure anonyme. Entièrement prises en charge par les religieuses, ces trois jeunes filles ont en commun d'avoir subi des agressions sexuelles commises par des femmes.

\subsection{Méthode et instruments de collecte des informations}

Deux méthodes ont été convoquées : l'étude de cas et la méthode clinique. Les techniques de recueil de données sont l'entretien clinique, le test du CD-RISC et l'Exercice de résilience (Strümpfer, 2001). Ajouter un test projectif comme l'Exercice de résilience au CD-RISC permet d'évaluer la résilience en explorant comment sont exprimés les facteurs de protection ; cela permet aussi de comprendre les réactions d'un individu face à une situation adverse. 
Le CD-RISC est une échelle de format Likert en cinq points, allant de 0 à 4 , le résultat final peut varier entre 0 et 100. Développée par Connor et Davidson (2003), elle comprend 25 items, lesquels sont répartis en cinq facteurs : le premier fait référence à la compétence personnelle, à un niveau d'exigence élevé et à la ténacité (huit énoncés) ; le deuxième correspond à la confiance en ses capacités, la tolérance aux émotions négatives et au renforcement des effets du stress (sept énoncés); le troisième concerne l'acceptation du changement et les relations interpersonnelles positives (cinq énoncés) ; le quatrième fait référence au sentiment de contrôle (trois énoncés) ; le cinquième est associé à la spiritualité (deux énoncés). Quant à lui, le test de l'Exercice de résilience (Strümpfer, 2001) comprend six situations auxquelles il faut imaginer comment l'individu peut faire face : deuil, perte d'emploi, difficulté financière, maltraitance, maladie physique et abus sexuel. Trois questions permettent de faciliter l'exploration de ce que le répondant imagine : que pense et ressent la personne ? Que va-t-il se passer? Plus tard, que signifiera pour la personne l'expérience vécue ? Les réponses permettent de comprendre comment l'individu réagit à l'adversité.

\subsection{Méthodes d'analyse et de traitement de données}

La méthodologie retenue est à la fois quantitative et qualitative. Les notes globales du CD-RISC ont été analysées conformément aux indications de Connor et Davidson (2003). L'exploitation de l'entretien clinique et de l'Exercice de résilience (Strümpfer, 2001) repose sur l'analyse des réponses du sujet. Dans le second test, les participantes ont été invitées à imaginer toutes les situations. La cotation repose sur le fait de se donner la résilience comme but, la signification, le soutien social auquel il fait appel ou qui est reçu. Nous adoptons donc la démarche suivante : vérification de la forme et du contenu de la réponse, des procédés utilisés et du processus de résilience pour chacun des six thèmes abordés.

\section{Résultats \\ 2.1. Cas 1 : Y. K. 13 ans \\ Âge au moment des faits : 12 ans \\ Lieu de l'agression : domicile familial \\ Durée de l'agression : 4 mois}

\subsubsection{Anamnèse :}

Y. K. a 13 ans. Elle est en classe de quatrième. Son père est décédé quand elle avait 4 ans et depuis lors, elle vivait chez sa grand-mère avec sa mère, sa tante et sa cousine germaine. Cette dernière, plus âgée, est en charge de veiller sur elle, lorsque les adultes sont absents. Les agressions sexuelles ont commencé après que Y.K. ait eu ses premières règles, sous la menace d'un 
couteau. La participante affirme qu'au début c'était des attouchements, puis des contacts bucco-génitaux. Ensuite, « elle a commencé à me toucher avec des objets, j'ai énormément saigné, c'est là que maman a su », dit-elle pour décrire l'agression sexuelle avec pénétration.

Le vécu de l'agression est marqué par des douleurs physiques, un malêtre et un sentiment de culpabilité « c'est pour ça que je n'ai rien dit à maman. J'avais peur, peur de maman même de Dieu, peur de Dani (c'est l'agresseure). Je ne sortais plus de la maison sauf pour aller prier avec mémé ». Il n'y a eu aucune plainte de la part de la mère. L'agresseur-e n'a donc pas été poursuivie en justice. Mais, elle a simplement été chassée de la maison, bannie de la famille. Y. K. n'a bénéficié d'aucun suivi psychologique. Sa grand-mère l'a conduite chez le pasteur de sa communauté pour des séances d'exorcisme «parce qu'elle avait peur que le démon qui habite ma cousine me possède aussi ... ça m'énervait d'y aller, je l'ai dit à mémé elle m'a dit que c'était le démon qui ne voulait pas prier et pas moi, donc elle allait m'obliger à partir. C'est pour cela que j'ai fui ». Se sentant coupable, elle a fugué. Elle est restée dans la rue pendant deux semaines et c'est ainsi qu'elle a rencontré les religieuses venues donner à manger aux sans-abris. "Les sœurs m'ont recueillie et sont allées rencontrer ma famille. Aujourd'hui, je préfère vivre au foyer. Je vais à l'école. J'ai de bonnes notes. J'ai terminé la première de ma classe, en cinquième. Mes profs m'apprécient et j'ai des amies. Ce n'est pas parce qu'une femme m'a fait ça que je suis lesbienne. J'ai tout le temps pour décider de qui je veux être. Je me sens bien ! ». Notons que Y.K. passe certains week-ends et les vacances dans sa famille.

\subsubsection{Résultat aux tests}

Au test du CD-RISC, Y. K. a obtenu 76 points, ce qui correspond au niveau moyen-supérieur de résilience (la moyenne est de 50 points). Elle est dont résiliente. Les résultats au test de l'Exercice de résilience montrent qu'elle considère l'adversité comme un défi dont la finalité serait une réussite sociale, laquelle passerait par la réussite scolaire.

\subsubsection{Analyse et interprétation}

Y. K. semble de prime abord une adolescence polie et respectueuse des normes. Elle se projette dans l'avenir. Elle veut devenir médecin. Elle reste persuadée que le traumatisme qu'elle a vécu est un défi de la vie et que « sa façon à elle de remporter le défi c'est de réussir ses études ». Ici, la résilience passe par la réussite scolaire. L'école est un facteur de résilience. C'est là un des éléments importants car Y. K se décrivait avant les événements comme une élève moyenne. Elle n'a pas su expliquer pourquoi elle travaillait peu avant et s'était mise à avoir de meilleurs résultats. On pourrait supposer que l'insuffisance de rendement était liée à l'environnement familial...On pourrait 
tout aussi bien, se dire qu'hors de cet environnement, l'agression sexuelle se révèle un mal pour un bien. La résilience de Y. K lui permet alors d'aller de l'avant...La réussite scolaire y est certainement pour quelque chose...mais quoi ? Au-delà de son rôle «d'ascenseur social », le contexte scolaire peut offrir à Y. K. une riche palette de ressources défensives et de possibilités de développer des supports de protection. Les facteurs de protection peuvent être basés sur les liens amicaux, les contacts positifs avec les adultes, la possibilité de réussir et d'être valorisé. On peut appréhender l'école comme un espace de protection pour Y. K. à plusieurs niveaux. En premier lieu, elle lui permet de développer ses compétences et de renforcer ses ressources propres. Le fait qu'elle soit première de sa classe semble avoir contribué à renforcer son estime de soi et sa confiance en soi. En second lieu, l'école peut être un cadre de protection susceptible d'atténuer les facteurs de risque issus de sa famille.

Dans les suites de l'agression sexuelle et de sa révélation, la religion apparait comme persécutrice et l'interprétation mystico-religieuse qui prévaut agit comme facteur traumatogène. La victime n'est pas entendue et elle est même aux bancs des accusés car, en refusant de prier, elle est coupable de collaboration avec la cousine et son démon (ce qui est l'explication plausible de cette passion masochiste). La bienveillance des religieuses agit à contrepied et ouvre à la possibilité d'une restauration psychique comme nous le voyons dans ce cas.

Le questionnement sur la féminité et l'identité sexuelle apparait à la lisière du discours «Ce n'est pas parce qu'une femme m'a fait ça que je suis lesbienne ». Cette phrase appartient-elle à Y. K ? N'est-ce pas un relent des perceptions mystico-religieuses familiales? Si ces questions ne semblent pas résolues pour l'instant, car elles ne constituent pas une priorité pour l'adolescente, elles sont essentielles dans le processus de l'adolescence. Les possibilités d'un suivi psychologique à long terme, devraient s'organiser autour de ces questions comme enjeux du développement psychoaffectif d'Y. K. Ici, «J'ai tout le temps pour décider de qui je veux être.» sonne alors comme un possible être à soi.

\subsection{Cas 2 : S. O., 15 ans}

Âge au moment des faits : 14 ans

Lieu : domicile familial de l'amie

Durée de l'agression : 1 jour

\subsubsection{Anamnèse :}

S. O. est une adolescente de 16 ans qui fait la classe de Seconde C (seconde scientifique). Née dans une famille musulmane, elle en a été chassée après l'agression sexuelle. Elle vit donc au foyer de jeunes filles auprès des religieuses de la communauté. Elle a été droguée puis agressée sexuellement 
par une de ses amies, pendant que le compagnon de cette dernière filmait les ébats. La participante affirme avoir été menacée: «ils ont menacé de divulguer les images à ma famille, dans mon école, si je ne leur donne pas l'argent que mon père cache dans son coffre. Je l'ai dit à papa. Il leur a donné de l'argent et m'a chassée de la maison. Il a dit que j'étais complice et que tout était de ma faute. » C'est sa mère qui l'a confié au foyer. Elle affirme qu'au début elle avait tout le temps peur, mais va mieux aujourd'hui. « Je me sens bien ici, je vais à l'école j'ai de bonnes notes, je vais à l'église maintenant, je prie et je demande à Dieu de m'aider à avancer. Je considère que mon passé ne détermine pas mon avenir. J'ai vécu quelque chose de difficile mais je m'en sors bien et c'est l'essentiel. Je sais que papa comprendra un jour. » Elle a toujours des contacts avec sa mère et ses frères. Pour S. O., le fait d'avoir été sexuellement agressée par une femme n'est pas différent d'une agression sexuelle commise par un homme. Elle ne se sent pas pour autant homosexuelle ou hétérosexuelle. Concernant le changement de pratique religieuse, elle dit : «j'accompagnais souvent mes amies à l'église avant l'agression, mais sans dire à ma famille. Au foyer je prie avec les sœurs... c'est venu comme ça... » Elle est convaincue que Dieu mettra sur son chemin la personne qu' il faut au moment opportun.

\subsubsection{Résultats aux tests}

S. O. a obtenu un score de 72 points au CD-RISC, ce qui la place nettement au-dessus de la moyenne. Le test de l'Exercice de résilience montre qu'elle perçoit la résilience comme une forme de compensation du traumatisme vécu. Elle fait référence à sa foi comme facteur de protection.

\subsubsection{Analyse et interprétation}

S. O. a de bons résultats scolaires et est admirée de ses enseignants qui la décrivent comme studieuse et exemplaire. Elle s'est décrite comme étant une élève moyenne, avant cette année scolaire. L'analyse de ses résultats aux tests montre qu'elle est résiliente. Elle fait souvent référence à ses croyances religieuses comme un fondement de cette reconstruction personnelle : "C'est Dieu qui m'aide et m'accompagne tous les jours ». Elle considère sa croyance comme une compensation au traumatisme vécu : «j'ai perdu un père, j'ai gagné un Dieu ». Cette façon de percevoir la religion comporte un facteur de résilience. En effet, S. O. est persuadée que sa capacité à surmonter le traumatisme vient de sa foi. Chez elle, la spiritualité donne « un accès » à un sentiment de transcendance, à un courage, une force qui aide et guide.

Aux yeux de son père, qui occulte l'agression sexuelle, S. O n'est pas une victime mais elle est complice du vol qu'il subit. Il la rejette. Sa mère échoue à la protéger et passe le relais à d'autres femmes. Si le Féminin doit s'interroger dans ce cas, tout comme le statut de mère, les questions qui 
semblent prioritaires sont celles en lien avec l'identité : identité sexuelle, identité religieuse, estime de soi, confiance en soi... Chez S. O, plus que la scolarité, c'est la pratique religieuse qui est un facteur avéré de résilience. La sublimation comme mécanisme de défense la protège des effets désorganisants du rejet familial que le changement de pratique religieuse rend encore plus problématique...Car, au final, que devra comprendre papa: qu'elle n'est pas complice du vol ? Qu'elle est victime d'agression sexuelle, de harcèlement et de chantage ? Ou bien que pour survivre, elle a choisi le dieu qui ne l'a pas jugée?

\subsection{Cas 3 de M. K., 17 ans}

Âge au moment des faits : 15 ans

Lieu de l'agression: domicile de la tante

Durée de l'agression : 1 jour

\subsubsection{Anamnèse}

M. K. est une orpheline de 17 ans qui fait la classe de seconde C. Elle vivait chez une tante à Abidjan, après le décès de ses parents dans un accident de la voie publique. Avant l'accident elle vivait en zone rurale au domicile de ses parents. Elle s'est enfuie de chez sa tante après l'agression sexuelle. Selon la participante, sa tante est une prostituée. Elle a abusé d'elle sexuellement à la demande d'un client. Elle n'a plus de contact avec cette dernière, mais elle garde le contact avec sa grand-mère maternelle restée au village. $M$. K. est restée dans la rue pendant un an, avant de rejoindre le foyer de jeunes filles, où elle vit. Aujourd'hui, elle poursuit sa scolarité normalement. Elle a un partenaire sexuel qui est dans le même lycée.

\subsubsection{Résultats aux tests}

M. K. a le score le plus élevé de résilience. Elle a obtenu un score de 82 points au CD-RISC. Cela pourrait s'expliquer par des facteurs comme : une confiance en soi, en ses compétences, à la valorisation de soi que le test de l'Exercice de résilience a fait ressortir. Ce test a mis en exergue les traits narcissiques de la participante comme facteur de résilience.

\subsubsection{Analyse et interprétation}

Le discours de M. K. est coloré de confiance en soi et de valorisation de soi : «Je suis devenue l'une des meilleures élèves de ma classe, les sœurs et les professeurs me citent en exemple ». À ce niveau, la confiance en soi est un sentiment fondé sur l'estime de soi, sur l'appréhension de sa valeur : «j'ai découvert que j'ai de la ressource », dit-elle avec humour. Ici, la confiance en soi peut être reconnue comme un facteur de protection face au traumatisme. Elle vient former un rempart contre l'adversité. M.K. ne se perçoit pas comme 
une victime mais plutôt comme une survivante. La question de l'identité sexuelle semble résolue. La participante se considère hétérosexuelle. Elle affirme: « le problème ne vient pas de moi, mais plutôt de ma tante. C'est elle qui va mal, pas moi. La sexualité est une bonne chose qui ne devrait pas être monnayée ». Elle se représente la sexualité de manière positive.

L'histoire de M. K. révèle non seulement un échec dans la protection de l'enfant, mais aussi les écueils du féminin. Sa tante brise la barrière maternelle protectrice en ayant une conduite incestueuse. D'où vient la force de cet enfant qui a perdu son enfance dans l'accident de ses parents, dans le déracinement et dans la trahison de la tante qui a abandonné la posture de protectrice pour se muer en persécutrice? Le niveau d'estime de soi engendrerait cette résilience particulière qui lui permet, sans difficulté majeure, de vivre son corps et son adolescence et de s'ouvrir à une sexualité partagée avec un homme sans question sur son identité sexuelle. Ni la religion, ni la scolarité n'apparaissent au-devant du tableau des facteurs de résilience ou tout au moins à minima.

\section{Discussion et Conclusion}

Ces trois vignettes cliniques ont mis en lumière, dans le sillage des agressions sexuelles commises par des femmes sur mineures : que l'agresseure était une personne de confiance ayant fonction de care (cousine, amie, tante). Aussi, la réaction de l'environnement perpétuait-elle la violence avec culpabilisation de la victime, rejet du milieu et contre-attitude de la victime qui fuyait le milieu ou choisissait une orientation de vie contraire aux choix familiaux. L'agression sexuelle qui a lieu à l'entame de l'adolescence a plus d'effet déstructurant sur l'identité sexuelle (YK et SO) que quand elle survient plus tard (MK). Cette violence perpétrée par des filles ou des femmes sur des filles questionne le féminin, l'identité et l'identité sexuelle/de genre. Cette problématique fera l'objet de recherches ultérieures.

Les facteurs individuels comme les compétences cognitives, les croyances religieuses et la confiance en soi sont déterminants dans l'analyse de la résilience chez les participantes. Ainsi, l'hypothèse générale est confirmée: les facteurs intra-individuels sont liés à la résilience chez les victimes d'agressions sexuelles commises par les femmes. Il faut noter qu'aucune des participantes n'a porté plainte pour agression sexuelle. Les agressions sexuelles des femmes sur les femmes sont peu documentées. Pour Sonia (2015), si la plupart de ces études soulignent un faible taux de femmes auteures de violences sexuelles, cela est probablement lié à l'image traditionnelle de la femme, perçue comme nourricière et protectrice, à la difficulté, pour les victimes, à dénoncer les agressions ou encore à la tendance sociale à minimiser ces délits. C'est ce qui s'est passé pour les trois adolescentes. Pour S. O. et M. K., l'agression a lieu en présence d'un tiers 
influenceur. L'agir sexuel violent des femmes agresseur-es, dans les vignettes cliniques que nous avons rapportées, semble porté par le désir de faire mal, de dominer...C'est une supposition car aucune des agresseur-es n'a été mise face à ses responsabilités ; aucune n'a été dénoncée. La non-dénonciation du crime et de la violence est potentiellement psychogène. Faire face au pouvoir destructeur et traumatogène de l'agression sexuelle, fait appel à la résilience des adolescentes.

Il est juste de penser que la résilience chez les victimes d'agression sexuelle passe par une possible réparation, par la condamnation ou par la judiciarisation de l'agresseur (Manciaux, 2005) mais ce n'est pas toujours le cas. En Côte d'Ivoire, peu de victimes portent plainte et peu d'agresseurs sont condamnés. Toutefois, des victimes surmontent leur traumatisme et parviennent à la résilience à l'image des participantes de la présente l'étude. Le construit de résilience pourrait revêtir un aspect culturel qui mérite qu'on s'y intéresse. Par ailleurs, les participantes à l'étude n'ont pas montré (au moment de l'étude) de trouble particulier lié à l'identité sexuelle. On peut prétendre que dans une agression homosexuelle, la représentation de la sexualité pourrait contribuer au processus de résilience chez les victimes. L'expérience de la sexualité est importante chez les adolescents, car ces premières expériences peuvent façonner la manière dont ils se représentent et vivent leur sexualité future. Les rapports sexuels exercés sous la contrainte sont plus susceptibles d'engendrer des expériences négatives. Ainsi, la sexualité peut être perçue comme une source de douleur. Or, M. K. donne une image positive de la sexualité. L'analyse des représentations ne constitue certes pas l'objet de la présente étude, mais le concept de résilience suppose une adaptation positive, dans le processus de protection. Aussi, la représentation positive de la sexualité pourrait-elle constituer une variable interne qui contribuerait à faciliter le processus de résilience.

Les observations faites dans la présente étude révèlent que les facteurs individuels comme la compétence cognitive (et la réussite scolaire), la spiritualité et la confiance en soi jouent un rôle dans le processus de résilience. Ces résultats rejoignent ceux de Sadia et Kouakou (2019), Ouatara et coll. (2014), ainsi que Kouakou-Mlan (2019). Les différences observées sont d'ordre méthodologique, notamment liées à la taille des échantillons. En effet, l'étude clinique de trois participantes ne permet pas de généraliser les résultats, même si cette méthode aurait l'avantage d'évaluer le fonctionnement psychique. Contrairement à l'étude de Sadia et Kouakou (2019), certains aspects de la personnalité, comme l'identité sexuelle, n'ont pas été examinés. Il aurait été intéressant d'observer la variabilité des représentations sexuelles chez les victimes d'agression homosexuelle, avec un échantillon plus grand.

Les questions relatives aux violences sexuelles sont encore taboues dans la société ivoirienne. On prétend souvent que les abus sexuels sur mineurs 
d'âge entraînent tôt ou tard des troubles de natures diverses, y compris des troubles de l'identité sexuelle. Le discours et le sens commun alimentent cette croyance ; ce qui conforte les victimes d'agression homosexuelle à garder le silence. Mais ces trois adolescentes au parcours de résilience atypique, laissent entendre que malgré les évènements de vie délétères et le traumatisme qu'elles ont vécues, la parole demeure la meilleure des armes contre les agressions sexuelles, qu'elles soient commises par un homme ou une femme. Les capacités individuelles dont elles font preuve sont des facteurs de protection contre l'adversité. L'institution en tant que lieu de vie, administrée par des femmes, redonne visage à la maternité, au féminin et au care. La bienveillance des religieuses qui transparaît dans les propos des adolescentes semblent les avoir réinscrites dans leur parcours existentiel. Sans toutefois nier la participation de l'institution qui les prend en charge, leur façon de percevoir le traumatisme et d'appréhender leur nouvelle vie met en lumière le rôle joué par les facteurs intra-individuels dans le processus de résilience.

\section{References:}

1. Anthony, E. J. (1982). L'enfant vulnérable. Presses Universitaires de France.

2. Béloudji, C., Le Masson, V., reyes, S. \& Bernard, G. (2017). Les violences contre les femmes et les filles et la résilience, Document de travail de Baced.

3. Bryden, D. \& Grier, M. (2011). The search of rapists' real motives. Criminology, 101, 1-15.

4. Cortoni, F., Hanson, R.K. \& Coache, M.E. (2009). Les délinquances sexuelles: Prévalence et récidive (Female sexual offenders: prevalence et recidivism). Revue internationale de criminologie et de police technique et scientifique, 62, 319-336.

5. Dagou, D. \& Goin Bi, Z. (2015). Les violences en milieu scolaire ivoirien. Perspectives psy, 54, 263-274.

6. Freeman, N.J. \& Sandler, J. C. (2008). Female and male sex offenders. Journal of interpersonal violence, 23 (10), 1934-1413.

7. Gabel, M. (2002). Les enfants victimes d'abus sexuels. Paris, PUF.

8. Garmezy, N. (1985). Stress-resistant children: the research for protective factors. Recent research in developmental psychopathology, 4, 213-233.

9. Garmezy, N. (1990). Resiliency and vulnerability to adverse with poverty. American behavioral scientist, 34 (4), 416-430.

10. Heim, C., Newport, D., Heit, S., Graham, Y., Wilcox, M., Bonsall, R. \& Miller, H. (2000). Pituatary-adrenol and automatic responses to stress in women after sexual and physical abuse in childhood. Psychiatry, 284, 592-597. 
11. Kouakou-Mlan, A.H. (2019). Traumatisme, résilience et réinsertion psychosociale des victimes de violences. Cas de six enfants en mobilité de l'association Jekawili de Bouaké. Thèse de Doctorat, Université Alassane Ouatara de Bouaké.

12. Koudou, O., Traoré, F. \& Bakayoko, I. (2015). La résistance au changement chez les femmes violentes en Côte d'Ivoire: Cas des exciseuses. European scientific journal, 11 (35), 222-238.

13. Lagrange, H. (1995). Les comportements sexuels des jeunes de 15 à 18 ans. La documentation française : Agence Nationale de Recherche sur le SIDA.

14. Le Pape, M. (2013). Viols d'hommes, masculinités et conflits armés. Cahiers d'Études Africaines, 209, 202-215.

15. Luissier, P. (2017). Les théories qui expliquent l'agression sexuelle de femmes. Traité de l'agression sexuelle, 1, 13-32.

16. Manciaux, (2005). Agression sexuelle et résilience. L'agression sexuelle coopérer au-delà des frontières, 1, 371-384.

17. Mbassa M.D. (2009). Chapitre 7, Les enfants victimes d'abus sexuels en Afrique in Regards d'Afrique sur la maltraitance, Karthala, 280 pages.

18. Ouatara, M., Koffi, K. J., Ballet, J. \& Mahieu, F. R. (2014). Résilience et équilibre en Côte d'Ivoire post-crise, Éthique et économique, 11 (1), 29-43.

19. Pascal, R. \& Hie, B. (2004). Le silence et la révélation : violences sexuelles et souffrance du dire, au risque du clivage. Cahiers de psychologie clinique, 2 (23), 59-79.

20. Rapport VACS-COTE D'IVOIRE. https://www.togetherforgirls.org/wpcontent/uploads/2020/10/RAPPORT-VACS-CI-FRANCAIS.pdf

21. Rutter, M. (1990). Psychosocial resilience and protective mechanisms, dans Rolf, J., Masten, D., Cicchetti, D., Nuechterlein, K.H. \& Weintraud, S. (1990). Risk and protective factors in the development of psychopathology, Cambridge University Press.

22. Sadia, M. \& Kouakou, H. (2019). Mobilité infantile en Côte d'Ivoire : facteurs de résilience et réinsertion psychosociale. International journal of multidisciplinary and current research, 7, 829-838.

23. Saradjn, J. (2010). Understanding the prevalence of female-perpetrated sexual abuse and the impact of that abuse on the victims. Dans Gannon, T.A. \& Cortoni, F., Female sexual offenders: theory, assessment, and treatment, Chichester, Wiley-Blackwell.

24. Sonia H, Vavassori D (2015). Les femmes auteures de violences sexuelles : étude clinique du parcours de vie et de la dynamique de l'agir sexuel violent. Bulletin de psychologie, 4,538, 319-330. 
25. Soutoul, PH. (1994). Les agressions sexuelles de l'adulte et du mineur, Paris, Ellipse.

26. Strümpfer, D. J. W. (2001). Psychometric proprieties of an instrument to measure resilience in adults. South Africa journal of psychology, 31(1), 36-44.

27. Tardif, M., Auclair, N., Jacob, M. \& Carpentier, J. (2005). Sexual abuse perpetrated by adult and juvenile females: an ultimate attempt to resolve a conflict associated with maternal identity. Child abuse and neglect, 29, 153-167. 